\title{
Efectividad de la Rehabilitación Fisioterapéutica, Ocupacional y Fonoaudiológica: Un Márco de Referencia (Segunda parte)
}

\author{
Alicia Trujillo Rojas $^{1} \quad$ Clemencia Cuervo Echeverri $^{2}$ Martha Escobar de Villate ${ }^{3}$
}

Este artículo es el segundo de dos publicaciones sobre la filosofía, la posicion teórica y el desarrollo operacional del Sistema EFECT: Efectividad de la Rehabilitación Fisioterapéutica, Ocupacional y.Fonoaudiológica. La primera parte se publicó en un número anterior de la revista Ocupación Humana ${ }^{1}$. Este sistema se creó, en la Universidad Nacional de Colombia, en respuesta a la carencia de un sistema uniforme para evaluar los resultados de los programas de rehabilitación. Este recurso responde a las exigencias actuales en materia de gerencia en salud, previstas en la legislación de seguridad social: documentación de resultados, monitoreo, manejo inteligente de la información, planeación y evaluación de programas ${ }^{2}$.

Con el propósito de facilitar la comprensión del Sistema, se ofrece información básica organizada en dos secciones: (1) el contexto para comprender el Sistema EFECT y (2) la presentación del sistema propiamente dicho.

\section{Contexto}

- Los conceptos y las definiciones de deficiencia, discapacidad y minusvalía según la Organización Mundial de la Salud (OMS).

- La prevalencia y las causas de la discapacidad.

- La política sobre discapacidad y rehabilitación.

- La comprensión moderna de rehabilitación como filosofía y como práctica.

- La identificación de sistemas internacionales para evaluar la efectividad de la rehabilitación en términos de desempeño funcional.

\section{Sistema EFECT}

- Concepto nuclear del Sistema EFECT : Desempeño Funcional para la Vida.

- Descripción del Sistema EFECT.

- Aplicación del Sistema EFECT.

- Formas de registro del Sistema EFECT (no se incluyen en este texto).

- Aciertos del Sistema EFECT.

\footnotetext{
${ }^{1}$ Terapeuta Ocupacional, Profesora Asociada Depto. de Terapias, Facultad de Medicina, Universidad Nacional de Colombia.

${ }^{2}$ Fonoaudióloga, Profesora Asociada Depto, de Terapias, Facultad de Medicina, Universidad Nacional de Colombia.

${ }^{3}$ Fisioterapeuta, Profesora Asistente Dpto. de Terapia, Facultad de Medicina, Universidad Nacional de Colombia.
} 


\section{Contexto}

\section{Conceptos y Definiciones de la OMS}

La OMS en 1980 propuso los conceptos deficiencia, discapacidad y minusvalía y su organización en un sistema ${ }^{3}$. La intención de este organismo internacional fue: (a) introducir conceptos y terminología no sólo para contemplar lo relativo a la enfermedad (deficiencia), sino también para asegurar la comprensión de sus consecuencias funcionales (discapacidad) y sociales (minusvalia); (b) orientar la formulación de política en la prestación de servicios de salud; (c) avanzar en la adopción de terminología uniforme para referirse a todas las condiciones discapacitantes y a sus consecuencias biológicas y sociales; (d) clarificar los alcances y la contribución de la atención médica, los servicios de rehabilitación y los servicios de bienestar social; (e) ofrecer criterios para la planificación de los servicios de salud; y (f) evaluar la disponibilidad, la eficiencia y los resultados de los programas de atención de salud. En su conjunto el sistema de clasificación fue pensado con el propósito de atenuar la visión limitada del modelo médico de la enfermedad:

El valor de la taxonomía de la OMS radica en la comprensión y categorización de las discapacidades como consecuencias de carácter funcional en el diario vivir de las personas (discapacidades) y en el reconocimiento de ladesventaja social (minusvalia) que obstaculiza el desempeño autónomo y realizanté de las personas en ambientes cotidianos.

Deficiencia tiene que ver con anormalidades de la estructura corporal y de la apariencia y con el funcionamiento de un órgano o sistema, resultantes de cualquier causa. En principio, las deficiencias representan trastornos en el nivel de los órganos. En el contexto de la experiencia de la salud, una deficiencia es la pérdida o anormalidad de la estructura psicológica, fisiológica o anatómica.

Discapacidad refleja las consecuencias de la deficiencia, en términos de desempeño funcional y actividad del individuo. Las discapacidades representan trastornos en el nivel de la persona. En el contexto de la experiencia de salud, una discapacidad es una restricción o falta de la habilidad para desempeñar una actividad de la manera o dentro del rango considerado normal para un ser humano, como resultado de una deficiencia.

La minusvalía está relacionada con las desventajas sentidas por el individuo, derivadas de las deficiencias y las discapacidades. Las minusvalías reflejan los obstáculos que se oponen a la interacción social y a la adaptación de la persona al ambiente que lo rodea. En el contexto de la experiencia de la sajlud, una minusvalía es una desventaja para un individuo, resultante de una deficiencia o una discapacidad, que limita o evita el cumplimiento de un desempeño que se considera normal para ese individuo, según edad, sexo y factores sociales y culturales.

\section{Prevalencia y Causas de la Discapacidad}

La discapacidad es una realidad mundial y una realidad colombiana. La OMS estima que el $10 \%$ de la población mundial tiene alguna forma de discapacidad. Esto significa que, por causa de deficiencias físicas, psíquicas o sensoriales, hay en el mundo 500 millones de personas con discapacidades, o sea una de cada 10 personas. 
Sin embargo, se estima que la existencia de la discapacidad involucra aproximadamente un $25 \%$ de los miembros de la sociedad para la realización de sus actividades productivas, si se tiene en cuenta no sólo a los discapacitados sino también a sus familiares y a todos aquellos que los asisten. ${ }^{4}$

En Colombia, el número de personas con discapacidades supera los 6 millones. En 1995, el Sistema Nacional dé Información sobre Discapacidad encontró que el $23.6 \%$ de los colombianos de nueve ciudades, estratos 1,2 y 3 , presenta alguna discapacidad. Esto significa que esta minoría asciende a 8.470.000. Sin embargo, como las consecuencias de la discapacidad también afectan al grupo familiar en sus relaciones emocionales y sociales y erosiona la productividad, la población vulnerada puede llegar a 15.000.000 de ciudadanos. ${ }^{5}$

Esta cifra tiende a aumentar debido a las condiciones de violencia y accidentalidad, trastornos cardiovasculares, morbilidad materno infantil, discapacidad infantil, consumo de sustancias tóxicas, alcoholismo y tabaquismo, envejecimiento de la población, desnutrición, maltrato infantil, estrés ocasionado por el estilo de vida, incremento de grupos marginales en condiciones de pobreza extrema y accidentes de trabajo. ${ }^{6}$ Esta alarmante situación exige que los programas de rehabilitación demuestren índices muy altos de efectividad.

La discapacidad es una realidad social grave, con consecuencias devastadoras que se aprecian en una pérdida significativa de años de vida saludable para los colombianos y en la disminución de la productividad en el país. El Banco Mundial y la OMS han cuantificado la pérdida de años de vida saludable no sólo con base en indicadores de mortalidad. Las sociedades también pierden años de vida saludable por incapacidad temporal o permanente.

En términos de la carga de la enfermedad en Colombia de los 5.512 .686 años de vida saludable (AVISA) perdidos en 1994, el $43 \%$ tuvo que ver con incapacidad temporal o permanente. De hecho, el Ministerio de Salud considera que el problema de la discapacidad en Colombia es un problema de salud pública creciente. En general, se ve sensiblemente afectado el indice de desarrollo humano del país.

\section{Política sobre Discapacidad y Rehabilitación}

Frente al problema de la discapacidad, las naciones del mundo formulan politica con el fin de asegurar el cumplimiento de los derechos ciudadanos de las personas con discapacidades y el aseguramiento de presupuesto para la planeación y ejecución de programas de servicios relacionados con la salud, el trabajo, la seguridad social, la vivienda, la educación, el transporte, la recreación, las telecomunicaciones y la rehabilitación. En síntesis, se legisla sobre la accesibilidad a los beneficios de la sociedad y la equiparación de oportunidades.

El siguiente es un recorrido que ilustra hitos importantes en la política internacional y nacional en materia de discapacidad y rehabilitación:

- Declaración Universal de los Derechos Humanos ( Naciones Unidas, 1948)

- Declaración Universal de los Derechos de las Personas con Discapacidad (Naciones Unidas, 1972).

- Carta para los Años 80 (Rehabilitación Internacional, 1980). 
- Declaración de Cartagena de Indias (Conferencia 'lberoamericana, 1992).

- Constitución Política de Colombia (1991).

- Ley General de Educación (Ministerio de Educación Nacional1993).

- Ley de Seguridad Social (Ministerio de Salud y Ministerio de Trabajo y Seguridad Social.1993).

- Manual Unico para la Calificación de la Invalidez (Ministerio de Trabajo y Seguridad Social: Decreto 692: de 1995).

- Plan Nacional de Atención a la Discapacidad (Vicepresidencia de la República, 1995).

- Clasificación Internacional de Deficiencias, Discapacidades y Minusvalias (OMS, 1980).

- Programa de Acción Mundial para las Personas con Discapacidades (Naciones Unidas, 1982)

- Convènio 159 sobre Readaptación Profésional y Empleo de Personas Inválidas (OIT, 1980).

- Política de Prevención y Atención a la Discapacidad (CONPES, 1995).

- Salud y Seguridad Social (CONPES, 1995).

- Plan Decenal de Educación (Ministerio de Educación Nacional, 1996).

- Ley de la República para el Fomento e Integración Social de las Personas con Limitación (1996).

\section{La Filosofía de la Rehabilitación: Comprensión Moderna}

Para enfrentar los problemas de discapacidad han surgido, en las sociedades contemporáneas, los programas de rehabilitación diseñados para promover a las personas con deficiencias y discapacidades, de manera que su desempeño físico, social y psicológico sea óptimo. Los programas de fisioterapia, terapia ocupacional y fonoaudiología constituyen la esencia de la rehabilitación. Se entiende por rehabilitación una respuesta social a là discapacidad. La respuesta incluye la expedición de leyes y la formulación de política; el desarrollo de la industria de la rehabilitación; la formación de profesionales de rehabilitación; el mercadeo de productos y servicios de rehabilitación; y la instauración de procesos de rehabilitación, o sea, el proceso de rehabilitarse en organizaciones de servicios humanos?

En lo que tiene que ver conexpedición de leyes, en Colombia ya existe la Ley de la República para el Fomento e Integración Social de las Personas con Limitación. En cuanto a formulación de política, el Ministerio de Salud, por la Resolución No. 03165 de 1996, declaró como de obligatorio cumplimiento para las entidades promotoras de salud y las instituciones prestadoras de servicios de salud unos lineamientos de atención para las personas con deficiencias, discapacidades y/o minusvalías. En lo que hace a laindustria de la rehabilitación, este desarrollo es incipiente en Colombia. La formación de profesionales de rehábilitación tiene una historia de aproximadamente cinco décadas. En lo que respecta al mercadeo de productos y servicios de rehabilitación, se puede decir que la 
gerencia moderna apenas empieza. Y por.último la instauración de procesos de rehabilitación institucional ha tenido una visión asistencialista y reduccionista.

En las sociedades contemporáneas, los sistemas de rehabilitación surgen del esfuerzo conjunto de muchas personas y sectores, dada la complejidad biológica, cognocitiva, emocional y sociocultural de las deficiencias, las discapacidades y las minusvalías. Por esto los procesos de rehabilitación comprometen a individuos, familias, administradores educativos y empleadores, entre otros. Además, incluyen una combinación de bienes, servicios y técnicas utilizados para ayudar al individuo a mejorar el desempeño autónomo y eficiente en ambientes naturales.

La rehabilitación asume un enfoque interprofesional ${ }^{8}$ frente a la deficiencia, la discapacidad y la minusvalia. Un grupo interprofesional debe incluir a: la persona con discapacidad, miembros seleccionados de la familia, un líder y profesionales de diferentes campos necesarios para una evaluación y tratamiento totalizante.

Este enfoque interprofesional es una estrategia gerenciada de toma de decisiones que facilita: el diseño de metas integradas a corto, mediano y largo plazo para lograr los mejores resultados, funcional y socialmente válidos para la persona con discapacidad; la coordinación de los programas de atención; la evaluación y el tratamiento simultáneo de desórdenes motores, sensoriales, cognoscitivos, emocionales y de habla y lenguaje; y el ajuste personalizado de los protocolos de intervención según las prioridades y las respuestas del usuario al tratamiento. El grupo debe ser dirigido por un gerente de caso $^{9}$. Este profesional debe tener habilidades clinicas y de gerencia en la administración de casos. Es responsable de administrar y coordinar los recursos y los servicios; facilitar el avance del usuario a través del proceso total de rehabilitación; asegurar la eficiencia y el rendimiento de cuentas; propiciar la toma de decisiones y la comunicación entre los miembros del grupo; y producir el plan de seguimiento para la transición de la persona a su ambiente natural.

La rehabilitación en Colombia, deberá superar la práctica de "trabajo en grupo" que ha prevalecido caracterizada, entre otros aspectos, por la visión fragmentada de la persona con discapacidad; el enfoque exclusivamente clínico; la ausencia de metas para la vida; la falta de concertación a favor del usuario, la asignación arbitraria de tareas, la ausencia de verdadero liderazgo; y la evasión de la responsabilidad individual con el pretexto de que existe un "grupo responsable".

En Colombia el análisis del impacto de la rehabilitación se ha efectuado sólo a través de la medición de resultados clínicos. Esta visión reducida no ha tenido en cuenta que el impacto de la rehabilitación se determina por su efectividad, o sea por la modificación positiva de seis factores: (a) la dimensión fiscal, por ejemplo los aportes que la persona rehabilitada revierte al Estado al reasumir su calidad de persona económicamente activa y la consecuente reducción de la inversión en seguridad social por régimen subsidiado; (b) los resultados clínicos o modificación de la condición biológica; (c) el estado de desempeño funcional o habilidad para actuar de manera independiente en ambientes naturales; (d) la satisfacción del consumidor o percepción del usuariø respecto a las ganancias obtenidas por el tratamiento; (e) la calidad de vida o condición tanto objetiva como subjetiva de 
bienestar; $y(f)$ las relaciones costo-efectividad o aumento de años de vida saludable con la menor inversión.

\section{Identificación de Sistemas Internacionales}

Internacionalmente, el desarrollo de sistemas operacionales para medir la efectividad de los procesos de rehabilitación ha alcanzado altos niveles de especialización ${ }^{10}$. La complejidad de estos sistemas va desde escalas y potentes bases de datos relacionales hasta sistemas expertos que apoyan la toma de decisiones referente a diversas dimensiones del proceso de rehabilitación. Algunos de estos sistemas internacionales para evaluar la efectividad de la rehabilitación son: elfunctional Independence Measure FIM ${ }^{11}$ o Medida de Independencia. Funcional, el Uniform Terminology o Terminología Uniforme ${ }^{12}$, el Functional Assessment of Communication Skills, ASHA FACS ${ }^{13}$ o Evaluación Funcional de Habilidades de Comunicación; el Occupational Therapy Assessment of Functional Activities OT FACT ${ }^{14}$ o Evaluación de Actividades Funcionales, y el Functional Status ${ }^{15}$ o Estado Funcional una medida empleada en fisioterapia.

\section{Presentación del Sistema EFECT}

Frente al reto que representa el diseño de sistemas que permitan documentar la efectividad de la rehabilitación, en la Universidad Nacional de Colombia Cuervo, Trujillo \& de Villate ${ }^{16171819}$, han venido investigando con el propósito de construir y validar un recurso conceptual y tecnológico que responda a esta exigencia. Este recurso se denomina Sistema EFECT: Sistema para Evaluar la Efectividad de la Rehabilitación Fisioterapéutica, Ocupacional y Fonoaudiológica.

\section{Concepto Nuclear}

El concepto nuclear del Sistema EFECT es el desempeño funcional para la vida, un nuevo término acuñado por Cuervo, Trujillo \& de Villate ${ }^{20}$, què sé refierè a la actuación de la persona con discapacidad en un escenario que: (a) sea sensible a las capacidades, idiosincracia y expectativas de la persona y su familia; (b) reconozca la eventualidad de limitaciones no reversibles; (c) ante las limitaciones permanentes, facilite el proceso de duelo y el cambio en la forma de vida de la persona con discapacidad; (d) logre un balance entre las capacidades y expectativas de la persona y las exigencias de la sociedad; (e) comprenda que la fusión del movimiento, la ocupación y la comunicación tiene un efecto exponencial en la calidad de vida de la persona; ( $f$ ) entienda el papel esencial que cumple la tecnología de rehabilitación en el logro de las metas de independencia; y (g) promueva la disminución de obstáculos físicos, sociales y legales, o sea que reduzca la minusvalia.

\section{Descripción del Sistema EFECT}

El Sistema está compuesto por dos dimensiones de efectividad: (1) los desempeños funcionales; $y$ (2) la percepción del usuario.

En cuanto a la Dimensión 1, el Sistema postula que una persona se mueve, se ocupa y se comunica en cuatro escenarios: en la casa, fuera de la casa, en el trabajo/estudio y con los amigos/se distrae. 
La dimensión describe cada escenario a través de 7 (siete) indicadores, para un total de 28 (veintiocho). Se relacionan con ejecuciones cotidianas, por ejemplo, en la casa una persona hace reparaciones, usa el teléfono ...; en el trabajo una persona soluciona problemas, maneja el estrés....

Cada uno de los siete indicadores se califica en tres momentos: primera evaluación, evaluación final y seguimiento. Se utiliza una escala de 4 grados de autonomía en la ejecución (sin ayuda de otros, con supervisión, con ayuda de otros y no. lo hace). La referencia para la evaluación de la efectividad del programa de rehabilitación es el cumplimiento de una meta negociada con el usuario y su familia. El sistema incorpora las dimensiones de: tecnología de rehabilitación y reducción de obstáculos físicos, sociales y legales. Sin estos requisitos no se puede hablar de efectividad de la rehabilitación fisioterapéutica, ocupacional y fonoaudiológica.

En relación a la Dimensión 2, el Sistema postula que el desempeño funcional no se puede apreciar exclusivamente desde la perspectiva de los profesionales de la rehabilitación. Es indispensable contar con la visión del usuario respecto a su realidad frente a la discapacidad, a la rehabilitación y a la vida: Por tanto, el Sistema EFECT identifica la visión del usuario en relación con el programa de rehabilitación, a través de su respuesta a 18 preguntas relacionadas con la discapacidad, la rehabilitación y la vida. Cada pregunta se califica en un rango de tres opciones cualitativas, a través de las cuales se establece si la satisfacción del usuario es positiva, parcial o negativa. Por ejemplo:

- El problema que tuvo cambió su forma de vivir?

- Ante ese cambio de vida, el programa de rehabilitación fue una ayuda para usted?

- Cómo ve su vida dentro de un año?

- Al comenzar su tratamiento negociamos las metas que usted podría lograr en un tiempo determinado. Cree que se logró lo acordado?

- El tratamiento tuvo en cuenta que usted necesitaba ayudas tecnológicas para mejorar su autonomía?

- Cree que el programa de rehabilitación contribuyó a disminuir algunos de los obstáculos que ha enfrentado a raiz de su problema... físicos, actitudinales, laborales?

- Lo que usted logró con el tratamiento le facilitó la vida a su familia?

\section{Aciertos del Sistema EFECT}

Sin duda, el Sistema EFECT representa un avance para la práctica de la rehabilitación en Colombia, en su filosofía, en su posición teórica y en su desarrollo operacional, por cuianto:

- Tiene en cuenta lineamientos nacionales e internacionales sobre discapacidad y rehabilitación.

- Contribuye a la validación de la clasificación de la OMS.

- Responde a las exigencias del Sistema de Seguridad Social en, Salud en materia de gerencia de servicios. 
- Incorpora el enfoque de trabajo interprofesional que utiliza la gerencia de caso como estrategia para asegurar la efectividad de lós programas de rehabilitación.

- Entiende que el proceso de rehabilitación busca el cumplimiento de metas sucesivas y alcanzables en el tiempo.

- Comprende que las metas de un programa de rehabilitación deben interpretar las necesidades más sentidas y vitales de la persona y su familia.

- Incorpora la participación del usuario y su familia en la toma de decisiones sobre metas y resultados.

- Valida la importancia del apoyo familiar.

- Promueve en la persona con discapacidad la planeación prospectiva con base en potenciales.

- Comprende los resultados de la rehabilitación en términos de la fusión de tres atributos esenciales de la vida: el movimiento, la ocupación y la comunicación.

- Entiende que la tecnología de rehabilitación es esencial en el logro del desempeño funcional.

- Comprende el impacto de los obstáculos físicos, actitudinàles y legales.

- Es amigable y fácil de utilizar.

\section{REFERENCIAS}

1 Trujillo, A.; Cuervo, C., de Villate, M. (1996). Efectividad de la Rehabilitäción Fisioterapeutica, Ocupacional y Fonoaudiológica: Un Marco de Referencia. Revista de Terapia Ocupacional. Santa Fe de Bogota. 6(3), 11-28.

2 Cuervo, C., Trujillo, A., de Villate (1.995). Efectividad de la Rehabilitación Fisioterapéutica, Ocupacional y Fonoaudiológica. Proyecto presentado a COLCIENCIAS. Santafé de Bogotá.

3 Organización Mundial de la Salud (1980). Clasificación internacional de deficiencia, discapacidad y minusvalía. Madrid, España.

4 Conferencia Intergubernamental Iberoamericana (1992). Declaración de Cartagena de Indias sobre Politicas Integrales para las Personas con Discapacidad en el Area Iberoamericana. Cartagena.

5 Ministerio de Salud (1995). Discapacidad y minusvalidez, situación, políticas y estrategias en Colombia. Documento multicopiado. Santafé de Bogotá: Ministerio de Salud.

6 Cuervo, C., Trujillo, A., de Villate, M. (1995a, julio). Comprensión Pública de la Discapacidad y la Rehabilitación. VII Congreso de Espécialidades Clínicas y Ciencias Básicas Asociación de Exalumnos de Medicina, Universidad Nacional de Colombia, Santafé de Bogotá.

7 Albrecht, G. L. (1992). The Disability Business. Newbury Park: Sage Publications:

CA 91320 
8 American Speech-Languaje-Hearing Association. (1995). Guidelines for the Strcture and Funtion of an interdisciplinary Team for Persons Whit Brain Injury.Asha, $37(3), 23-25$.

9 Hettinger,J. (1996). Case Management: do Ots have what it takes? OT.Week, $10(31), 12-14$.

10 Cuervo, C., Trujillo, A., de Villate, M. (1993) Modelo básico de información en rehabilitación (MBIR). Revista de la Facultad de Medicina, Universidad Nacional de Colombia. 41(3), 131-134.

11 Granger, CV y Hamilton, BB (1994). The uniform data system for medical rehabilitation report of first admissions for 1992. American Journal of Physical Medicine and Rehabilitation, 51-56.

12 American Occupational Therapy Association (1989). Uniform Terminology for Occupational Therapy - Second Edition. American Journal of Occupational Therapy, 43 (12), 808-815.

13 American Speech-Language-Hearing Association(1995). ASHAFACS. A Functional Outcome Measure for Adults. Rockville, MD: El Autor.

14 Smith, R.O. (1990). Administration and Scoring Manual. OT FACT (Occupational Therapy Functional Assessment Compilation Tool) Rockville, Maryland: TheAmerican Occupational Therapy Association, Inc.

15 Monahan, B. (1995). Managing under managed care. PT . Magazine of Physical Therapy, 3, 7, 38-47.

16 Cuervo, C., .Trujillo, A., de Villate, M. (1993) Modelo básico de información en rehabilitación (MBIR). Revista de la Facultad de Medicina, Universidad Nacional de Colombia. 41(3), 131-134.

17 Cuẹvo, C., Trujillo, A., de Villate, M: (1995a, julio). Comprensión Pública de la Discapacidad y la Rehabilitación. VII Congreso de Especialidades Clínicas y Ciencias Básicas Asociación de Exalumnos de Medicina, Universidad Nacional de Colombia, Santafé de Bogotá.

$18 \cdot$ Cuervo, C., Trujillo, A., de Villate (1995b). Comprensión Pública de la Discapacidad. Revista de la Facultad de Medicina de la Universidad Nacional de Colombia. Santafé de Bogotá. En prensa.

19 Cuervo, C., Trujillo, A., de Villate (1995). Efectividad de la Rehabilitación Fisioterapéutica, Ocupacional y Fonoaudiológica. Proyecto presentado a COLCIENCIAS: Santafé de Bogotá.

20 Trujillo, A., Cuervo, C., de Villate, M. (1996). Efectividad de la Rehabilitación Fisioterapeutica, Ocupacional y Fonoaudiológica: Un Marco de Referencia. Revista de Terapia Ocupacional. Santa Fe de Bogota. 6(3), 11-28 